\title{
Apathy in Alzheimer's Disease: Any Effective Treatment?
}

\author{
Raffaele Rea, ${ }^{1,2}$ Anna Carotenuto, ${ }^{1,2}$ Angiola M. Fasanaro, ${ }^{2}$ \\ Enea Traini, ${ }^{1}$ and Francesco Amenta ${ }^{1}$ \\ ${ }^{1}$ Centro di Ricerche Cliniche, Telemedicina e Telefarmacia, Università di Camerino, 62032 Camerino, Italy \\ ${ }^{2}$ Unità Complessa Neurologia, Azienda Ospedaliera di Rilievo Nazionale A. Cardarelli, 80131 Naples, Italy \\ Correspondence should be addressed to Francesco Amenta; francesco.amenta@unicam.it
}

Received 21 August 2013; Accepted 6 November 2013; Published 2 February 2014

Academic Editors: C. Capurso, P. Dal-Bianco, and N. Tabet

Copyright (C) 2014 Raffaele Rea et al. This is an open access article distributed under the Creative Commons Attribution License, which permits unrestricted use, distribution, and reproduction in any medium, provided the original work is properly cited.

\begin{abstract}
Objective. This review has evaluated the effectiveness of pharmacological treatment of apathy in patients with Alzheimer's disease (AD). Methods. A systematic literature search was conducted on published clinical trials assessing the effects of pharmacological treatment on apathy in $\mathrm{AD}$ over the last 10 years. Results. Fourteen studies considered of good quality were included in the analysis (4 randomized controlled trials, 9 open-label studies, and 1 retrospective analysis). Cholinesterase inhibitors were investigated in 9 studies, monoaminergic compounds such as methylphenidate and modafinil in two trials and one trial, respectively, and Ginkgo biloba (EGb 761 extract) and citalopram in one study each. Cholinesterase inhibitors did not show statistical significant effect in 1 RCT study but were associated to improvement in 3 open-label studies. Methylphenidate elicited a small but significant activity accompanied by relevant side effects such as high blood pressure, cough, and osteoarticular pain. EGb 761 was well tolerated and countered apathy. Other treatments induced modest improvements or were ineffective. Conclusions. Apathy treatment remains a challenge and there is no evident advantage of any specific pharmacotherapy tested so far. The development of controlled studies according to updated guidelines for the diagnosis of apathy in patients with $\mathrm{AD}$ is desirable.
\end{abstract}

\section{Introduction}

Apathy is a neurocognitive disorder characterized by the reduction of goal-directed behaviour. It may be found in different diseases and is common in neurodegenerative disorders. In Alzheimer's disease (AD) it has been found to be associated with specific atrophy patterns [1]. The first definition of apathy came in 1990 by Marins [2], who classified it as a motivational loss not attributable to emotional stress, cognitive impairment, or consciousness reduction [3]. Apathy has been subsequently related to disconnection of different brain circuitries, including the amygdala, which is innervated by cholinergic projections from the basal forebrain [4], thalamic nuclei of the midline, the basal forebrain, and the cholinergic pedunculopontine projections. The predominant cholinergic nature of nuclei probably disconnected in apathy suggests that impaired brain cholinergic neurotransmission is involved in apathy pathophysiology.

Recently apathy was classified from a clinical point of view into three different subtypes, namely, emotional affective apathy, cognitive apathy, and autoactivation apathy. Each of these forms of apathy is related to the impairment of specific processes [5]. Apathy due to the disruption of the "emotionalaffective" processing is attributed to damage of circuits linking emotional-affective signals to ongoing or forthcoming behaviors. Lesions of the orbitomedial prefrontal cortex and/or related regions (limbic territory) of the basal ganglia (ventral striatum, ventral pallidum) are probably linked to this form of apathy. Apathy due to the disruption of the "cognitive processing" is attributed to impairment of circuits linking the planning of actions to the ongoing or forthcoming behaviors. Lesions of the dorsolateral prefrontal cortex and/or related regions (associative territory of the basal ganglia including the dorsal caudate nucleus) might be responsible for it. Apathy due to the disruption of "autoactivation" processing is attributed to damage of the circuits linking the selfactivation of thoughts and actions to ongoing or forthcoming behaviors. Externally driven behaviors are relatively unaffected in autoactivation apathy. Lesions affecting bilaterally associative and limbic territories of the internal portion of 
the globus pallidus might be responsible for it [5]. From an epidemiological point of view apathy can be considered as a common symptom of AD. The frequency of it ranges from 19 to $76 \%[6]$, depending on the disease duration and stage and the age of subjects $[7,8]$.

The European Alzheimer's Disease Consortium has issued in 2008 guidelines for apathy diagnosis [9]. According to these guidelines for a correct diagnosis of apathy the diminished motivation clinical picture must persist for no less than four weeks, and two of the following three dimensions should be present: (i) reduced goal-directed behavior, (ii) reduced goal-directed cognitive activity, and (iii) reduced emotions. Moreover, functional impairment should be attributable to apathy [10]. Apathy is the cause of high distress levels in caregivers [11, 12], as they need to take continuous responsibility for everything. Over time, anger and conflicts inevitably follow between patients and caregivers. This makes apathy a risk factor for institutionalization.

Diagnostic Criteria for Apathy Revised by European Alzheimer's Disease Consortium (EADC). For a diagnosis of apathy the patient should fulfill criteria A, B, C, and D.

Criterion A. Loss of or diminished motivation in comparison to the patient's previous level of functioning is not consistent with his age or culture. These changes in motivation may be reported by the patient himself or by the observations of others.

Criterion $B$. It signifies the presence of at least one symptom in at least 2 of the 3 following domains for a period of at least 4 weeks and present most of the time.

B1. Domain Behaviour. Loss of or diminished goal-directed behaviour is evidenced by at least one of the following:

(i) initiation symptom: loss of self-initiated behaviour (e.g., starting conversation, doing basic tasks of dayto-day living, seeking social activities, and communicating choices);

(ii) responsiveness symptom: loss of environment-stimulated behaviour (e.g., responding to conversation and participating in social activities).

B2. Domain Cognition. Loss of or diminished goal-directed cognitive activity is evidenced by at least one of the following:

(i) initiation symptom: loss of spontaneous ideas and curiosity for routine and new events (i.e., challenging tasks, recent news, social opportunities, and personal/family and social affairs);

(ii) responsiveness symptom: loss of environment-stimulated ideas and curiosity for routine and new events (i.e., in the person's residence, neighborhood, or community).

B3. Domain Emotion. Loss of or diminished emotion is evidenced by at least one of the following:

(i) initiation symptom: loss of spontaneous emotion, observed or self-reported (e.g., subjective feeling of weak or absent emotions, or observation by others of a blunted affect);

(ii) responsiveness symptom: loss of emotional responsiveness to positive or negative stimuli or events (e.g., observer reports of unchanging affect or of little emotional reaction to exciting events, personal loss, serious illness, and emotional-laden news).

Criterion C. These symptoms (A and B) cause clinically significant impairment in personal, social, occupational, or other important areas of functioning.

Criterion D. The symptoms (A and B) are not exclusively explained or due to physical disabilities (e.g., blindness and loss of hearing), to motor disabilities, to diminished level of consciousness, or to the direct physiological effects of a substance (e.g., drug of abuse and a medication).

The purpose of this work was to review the effectiveness of pharmacological treatments for apathy in AD. Efficacy, adverse effects, and specific indications of drugs investigated in clinical trials were analyzed comparatively.

\section{Methods}

A systematic literature search was conducted on the PubMed database. Analysis included papers (clinical trials) published from 2003 to 2013 using the following entries: "apathy in AD" "pharmacological treatment of apathy in AD," "drugs for apathy in AD," and "medication for the treatment of apathy in AD." For the entry "apathy in AD" 57 papers were identified, for the entry "pharmacological treatment of apathy in $\mathrm{AD}$," 24 studies were found, for the entry "drugs for apathy in $A D$ " 4 papers were identified, and for the entry "medications for the treatment of apathy in AD", 3 studies were found. A total of 88 papers were identified and 14 of them were selected for further evaluation as they fulfilled the "good quality" criteria detailed below. Criteria for inclusion of an article in our review were as follows: (a) the study population should involve patients with $\mathrm{AD}$; (b) the study objective has to evaluate the effects of a pharmacological treatment; (c) apathy represents the primary or secondary outcome of the investigation; (d) the article should be in English; (e) abstract is present; (f) the term "apathy" should be present in the title/abstract (g). Randomized controlled clinical trials (RCTs) were included, whereas review articles were excluded. For nonrandomised studies, the Newcastle-Ottawa Scale criteria that enable to assess quality studies with their design were used [27].

\section{Results}

Treatment of apathy in $\mathrm{AD}$ has been evaluated as a primary outcome in 8 studies and as secondary outcome in 6 trials. Collectively the present review has considered 4 RCTs, 9 open-label studies, and 1 retrospective analysis (Tables 1,2 , and 3). Apathy was often assessed as a part of neuropsychiatric symptoms using items such as the Neuropsychiatric Inventory (NPI), the Clinical Global Impression of 


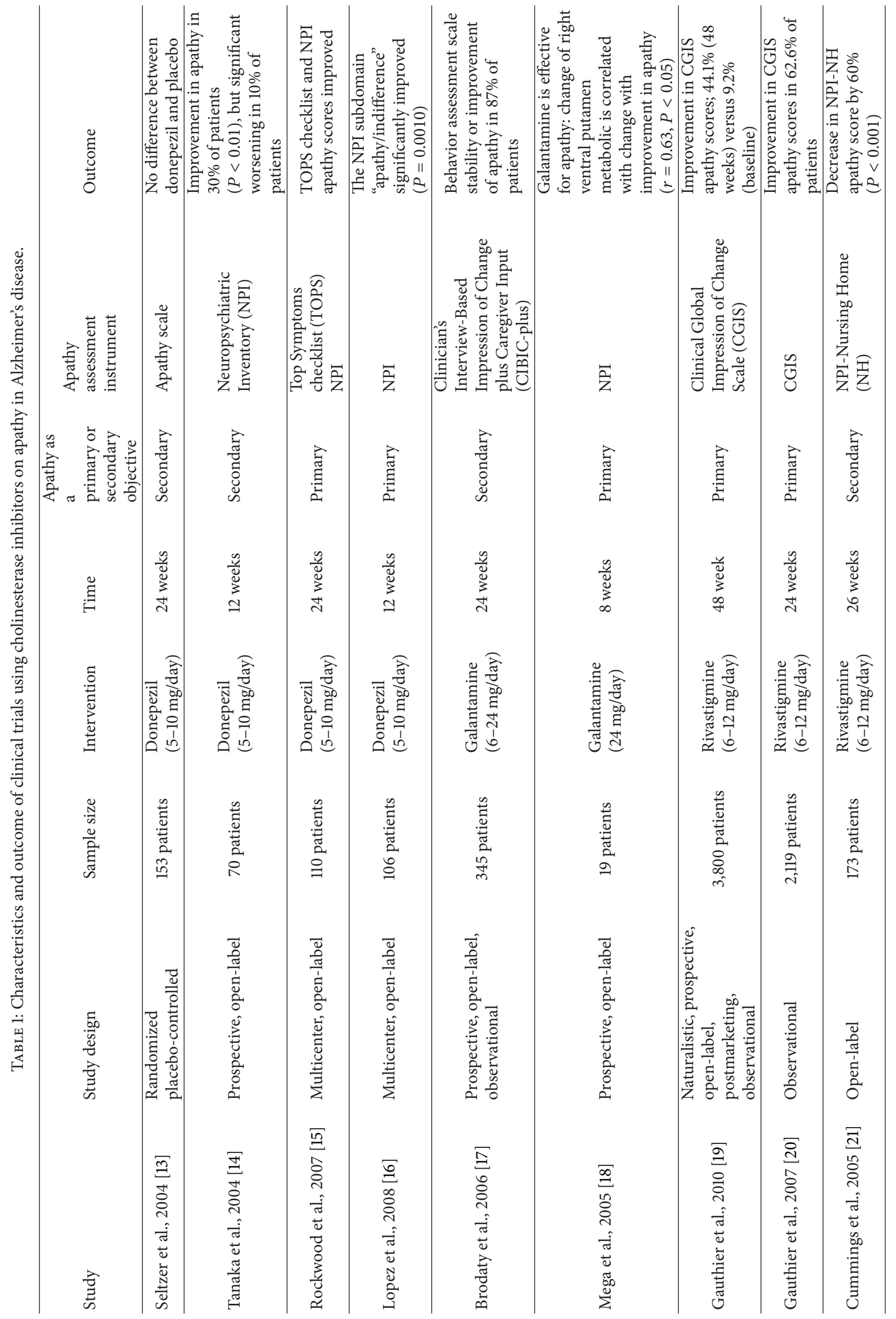




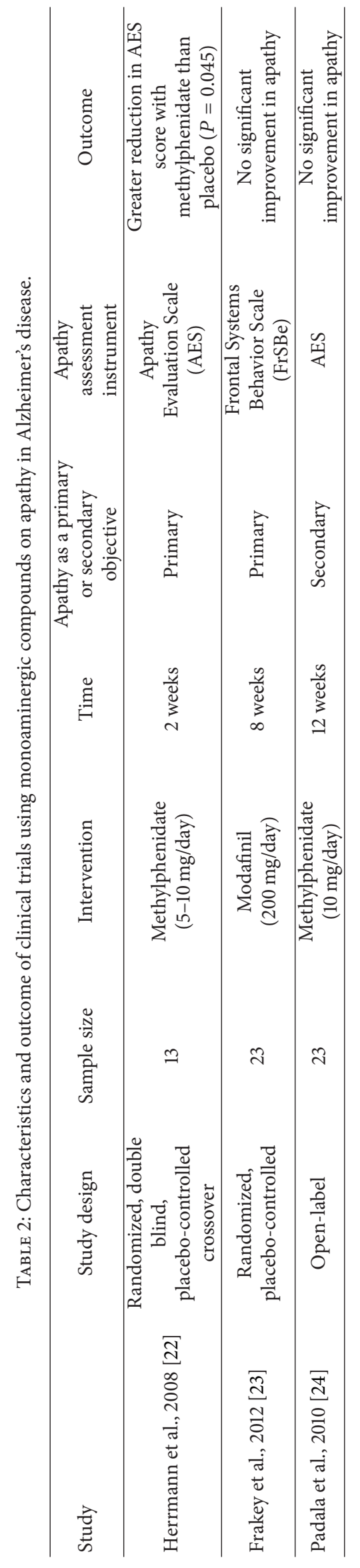




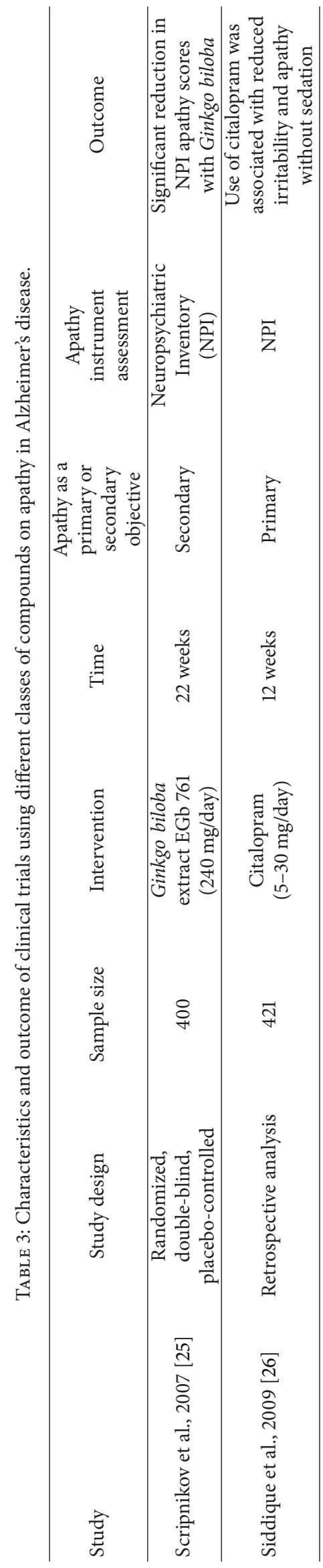


Change (CGIC) Scale, the Clinical Scale of Neuropsychiatric Symptoms (TOPS), and the Frontal Systems Behavior Scale (FrSBe). A specific questionnaire for apathy, such as the Apathy Evaluation Scale (AES), was used only rarely.

3.1. Randomized Controlled Clinical Trials. Of the 4 studies retrieved, 2 assessed apathy as a primary outcome and 2 as a secondary outcome. Among the cholinesterase inhibitors only one study has evaluated the efficacy of donepezil taking apathy as a secondary outcome in patients with $\mathrm{AD}$ at an early stage [13]. This work included 153 patients, 96 of whom were treated for 24 weeks with donepezil $(5 \mathrm{mg}$ /day in the first 6 weeks and $10 \mathrm{mg} /$ day thereafter). In the active treatment group a higher score at the scale of the apathy compared to placebo was reported. Differences among the two groups investigated were not significant [13]. In this study, treatment with donepezil improved memory tasks, attention, and cognition. Serious adverse events occurred in a similar number of donepezil- and placebo-treated patients [13] (Table 1).

The monoaminergic psychostimulant methylphenidate was effective in apathy in an RCT made in 2008 [22]. The study showed that 13 AD subjects treated with methylphenidate for 2 weeks ( 5 and $10 \mathrm{mg}$ thereafter) had a greater improvement at the AES total change scores compared with $12 \mathrm{AD}$ patients receiving placebo (end of treatment baseline; Wilcoxon $Z=-2.00, P=0.045)$. The NPI apathy scale too revealed a significant difference in scores between methylphenidate and placebo but with less effect (end of treatment baseline; Wilcoxon, $P=0.082$ ). Response to methylphenidate was also associated with increase in inattention to a continuous performance task after dextroamphetamine challenge [22]. Adverse events reported were delusions, agitation, irritability, and insomnia [22] (Table 2).

Effects of treatment with Ginkgo biloba on apathy were investigated in $400 \mathrm{AD}, \mathrm{AD}$ with cerebrovascular disease, and vascular dementia patients treated for 22 weeks with the EGb 761 extract of Ginkgo biloba [25]. Subjects treated with EGb 761 showed a significant improvement $(P<0.001)$ at the mean composite score of NPI frequency $\times$ severity (21.3 to 14.7 versus 21.6 to 24.1 ) and the mean score of the NPI caregiver distress (13.5 to 8.7 versus 13.4 to 13.9 ) [25]. The largest drug-placebo differences in favor of EGb 761 were found for apathy/indifference (statistical significance not reported) (Table 3 ).

One study has examined the efficacy of the monoaminergic agent modafinil (200 mg/day) on apathetic symptomatology in $23 \mathrm{AD}$ patients treated with cholinesterase inhibitors (Frakey, 2012) [23]. After 8 weeks of treatment, both the experimental (cholinesterase inhibitor + modafinil) and control (cholinesterase inhibitor + placebo) groups showed reductions in apathy on the Frontal Systems Behavior Scale between baseline and final assessments $(F=18.017, P<0.001$, $\left.\eta^{2}=0.474\right)$ [23]. No significant additional reduction in apathy was found with modafinil $\left(F=0.008, P=0.932, \eta^{2}=\right.$ $0.000)$. The addition of modafinil to the standard treatment for $\mathrm{AD}$ (cholinesterase inhibitor medication) did not result in significant additional reductions in apathy [23] (Table 2).
3.2. Retrospective Analysis. A retrospective study published in 2007 has investigated the efficacy of citalopram versus the antipsychotic drugs risperidone, olanzapine, quetiapine, and placebo in analyzing the database of CATIE (Clinical Antipsychotic Trials of Intervention Effectiveness in AD) trial [26]. Using data obtained with NPI score, apathy and irritability were investigated in 34 nondepressed AD subjects of the CATIE study. Patients were treated with placebo for 14 days and then with citalopram 5-30 mg for a minimum of 2 weeks and a maximum of 12 weeks (Table 3 ). Citalopram improved apathy and lowered irritability in the $60 \%$ of subjects, but the results were not statistically significant [26] (Table 3).

3.3. Open-Label Studies. The effectiveness of donepezil was demonstrated by 3 open-label studies performed including in total 186 patients treated for a period of 12-24 weeks. Apathy was evaluated by NPI or TOPS as a primary outcome measure [14-16] (Table 1).

A study on the prediction of psychiatric response to donepezil (5-10 mg/day) in patients with mild-to-moderate $\mathrm{AD}$ has shown that after 12 weeks of treatment the $30 \%$ of patients (21 of the 70 enrolled) showed a statistically significant improvement of behavioral disorders (primarily dysphoria, anxiety, and apathy) [14]. The $60 \%$ of patients did not respond to the treatment and the remaining $7 \%$ had a score statistically worse, particularly in the domain of apathy [14]. The three groups examined did not differ significantly at the baseline in age, education, MMSE, ADAS-cog (Alzheimer's Disease Assessment Scale-cognitive subscale), and Geriatric Depression Scale scores. Statistical parametric mapping analysis of single-photon emission computed tomography (SPECT) images at baseline showed that cerebral blood flow in the premotor and parietotemporal cortices was significantly higher in the responder group than in the worse group [14] (Table 1).

A multicentre study [15] has assessed specific symptomatic changes, including cognitive function, domestic activities, and neuropsychiatric disorders, through a symptom checklist of TOPS. Analysis was completed independently by caregivers and clinicians. The TOPS checklist consists of 19 items and includes a subitem for the evaluation of the overall impression on the symptom apathy. The study has compared neuropsychiatric and behavioral changes for 24 weeks in 101 AD patients treated with donepezil 5$10 \mathrm{mg} /$ day. Apathy was related to changes in standardized scales of cognition (ADAS-cog), activities of daily living (ADL), behavior (NPI), and caregiver burden (CAS). Caregiver evaluation reported that symptoms most improved were cognitive activation, attention, and apathy. Clinician assessments reported that attention was the symptom most often benefiting from treatment followed by apathy. The results at the TOPS checklist correlated significantly with those to standardized measures of cognitive function (ADAS-cog > $50 \%$ of the symptoms) (Table 1 ).

A study of effectiveness and safety in Hispanic patients [16] has evaluated the efficacy of donepezil (5-10 mg/day) in $106 \mathrm{AD}$ patients for 12 weeks. The mean NPI total score 
was statistically improved from baseline at 6 weeks $(-3.2$ points, $P=0.0018$ ) but not at 12 weeks. The NPI subdomain for apathy/indifference showed a statistically significant improvement from baseline at 12 weeks of treatment $(P=$ 0.001) (Table 1).

Two studies with galantamine $[17,18]$ have evaluated 364 patients for a period of 8-24 weeks. The assessment of apathy was carried out by NPI or CIBIC-plus [17] (Table 1). An observational study, the objective of which was to collect descriptive data on the treatment with galantamine $24 \mathrm{mg} /$ day under naturalistic conditions, has investigated the apathy domain [17]. Most subjects showed no deterioration in behavioral assessments over 3 and 6 months. At 6 months, the majority of patients either improved (range 7.3-28.0\%) or remained stable (range 58.7-84.0\%). The study did not show statistically significant changes for the evaluation of apathy with behavioral questionnaire CIBIC-plus [17] (Table 1).

Metabolic patterns associated with neuropsychiatric clinical response were investigated in a sample of $19 \mathrm{AD}$ patients [18] treated for weeks with $24 \mathrm{mg} /$ day galantamine. Six patients were classified as responders (NPI score improvement of at least 4 points), 7 did not change, and 6 were classified as nonresponders. After treatment, a significant increase of metabolism was found in the right thalamus of responders. Compared with their baseline values responders showed after treatment with galantamine an increase of the left caudate-thalamofrontal circuit metabolism. In both responders and nonresponders changes in the right ventral putamen were significantly correlated $(r=0.63 ; P<0.05)$ with improvement in apathy [18] (Table 1).

The activity of rivastigmine was investigated in three open-label studies [19-21] examining on the whole 6,752 patients treated for 24-48 weeks. Apathy was evaluated by Clinical Global Impression of Change (CGI-C) or the NPI score. In the first of these trials effects of rivastigmine were assessed in $173 \mathrm{AD}$ patients resident in a nursing home [21]. Efficacy of drug treatment was evaluated using the NPINursing Home (NPI-NH) test. At 26 weeks, an improvement of the scores for 8 of the 12 neuropsychiatric and behavioral disturbances in patients with specific apathy symptomatology at the baseline was noticeable [21]. The improvement from baseline of the apathy/indifference subitem of NPI-NH was statistically significant $(n=37 P<0.001)$. In the second of these trials [20], 2,119 $\mathrm{AD}$ patients were treated with rivastigmine (dose at the discretion of the prescribing physicians, 6 , 9 , or $12 \mathrm{mg} /$ day) for 6 months (Table 1). The results obtained reported an improvement of apathy in approximately $62.6 \%$ of patients treated, whereas attention, anxiety, and agitation ameliorated in $67.5 \%, 62.3 \%$, and $56.0 \%$ of patients, respectively (Table 1). In an another more recent and larger multicenter study, apathy was evaluated in 4,460 AD patients treated with rivastigmine 6-9-12 mg/day for 48 weeks [19]. Effects of treatment were assessed by the CGI-C taken as the primary outcome measure [19]. The authors' conclusion was that for each symptom investigated, the percentage of patients experiencing an improvement was greater than the percentage of patients with worsening of symptomatology. Apathy evaluation in eligible patients resulted in the proportions of deterioration improvement/worsening 42.8 versus $7.2 \%$ at 24 weeks and 44.1 versus $9.2 \%$ at 48 weeks [19] (Table 1).

The effectiveness of methylphenidate was demonstrated by a recent open-label study examining 23 patients [24]. The study has shown a significant improvement of apathy, assessed as primary outcome with the AES $(P<0.001)$ and NPI (statistical significance not reported). Adverse events such as increased blood pressure, hacking cough, dizziness, restless legs, sores in the mouth, arthritic pain in knuckles, and behavioral disturbances consisting of irritability, insomnia, and appetite change were reported. Some of these adverse events disappeared after decreasing methylphenidate from $10 \mathrm{mg}$ twice a day to $5 \mathrm{mg}$ twice a day.

\section{Discussion}

Apathy accompanies a broad range of central nervous system disorders, but its epidemiology, pathogenesis, and treatment options are not clear and often controversial $[5-8,28]$. This review on therapeutic strategies against apathy in $\mathrm{AD}$ showed that four categories of drugs were used for treating apathy. These include cholinesterase inhibitors, monoaminergic agents (methylphenidate and modafinil), and other different pharmacological profile drugs such as the Ginkgo biloba extract Egb 761 and the selective serotonin reuptake inhibitor (SSRI) citalopram. Among treatments compared, modafinil was ineffective and citalopram induced improvements that did not achieve a statistical significance.

Cholinesterase inhibitors were evaluated by 9 studies ( 1 RCT and 8 open label). These trials did enroll on the whole 7,655 patients, 6,752 of whom received rivastigmine. The global number of patients treated with cholinesterase inhibitors is certainly high, but not the time of evaluation that did not exceed the 6 months. The only RCT study with donepezil showed some nonstatistically significant improvement in the active treatment group. Among the 8 openlabel studies with this class of compounds, only 2 reached levels of statistical significance achieved with a $P<0.001$ in patients treated with rivastigmine [21] and a $P=0.001$ in donepezil-treated AD patients [14]. On a rather large sample of patients treated with cholinesterase inhibitors $(n=7,655)$ treatment induced a statistically significant improvement on apathy scores in only 379 (4.95\%) patients.

Methylphenidate has been evaluated in 2 studies: 1 RCT and 1 open-label study, enrolling only 36 patients, evaluated for 7 weeks. Effects of pharmacological treatment were significant in the two studies under discussion (RCT study, $P=0.045$ [22] and open-label study, $P<0.001$ [24]). Apart from the limited size of the sample, it should be pointed out that the best effects were seen with the dose of $10 \mathrm{mg}$ twice a day that was decreased due to serious adverse events elicited by treatment $[22,24]$. The Ginkgo biloba extract Egb 761 was evaluated in 1 RCT study on $400 \mathrm{AD}$ patients treated for 22 weeks. It induced a statistically significant improvement $(P<0.001)[25]$ and was well tolerated with adverse events less than those observed in patients treated with placebo in the same trial [25]. 
Comparing clinical studies reviewed here, the largest sample in which pharmacological treatment induced a statistically significant effect was that of EGb761 with 400 patients investigated, a number too limited considering the large population of AD suffering from apathy. Besides the quantitatively limited evidence of an activity of compounds tested, it should be pointed out that the majority of available studies have several limitations. First, apathy was diagnosed through the reliable AES scale only in 3 studies. This is a relevant issue, as the different components of apathy (emotional, cognitive, and autoactivation) can be addressed only by a specific tool such as AES that is different from the NPI and guarantees a correct differential diagnosis. Second, only a few studies have examined patients with enough characterization and in which cognitive decline was measured and indicated. Third, only a few studies have evaluated the presence of depression, which could have a symptomatic overlap with apathy and may be improved by appropriate treatment with antidepressants. Fourth, only one study has evaluated the neuropsychological functions connected with apathy and in particular executive functions to which apathy is attributed [14]. Executive functions should improve parallel to apathy improvement [14]. Fifth, measurement of effectiveness of pharmacological treatment was done using too many heterogeneous tools such as NPI, AES, and CIBIC-plus and other less common test batteries. This limits the possibility of a comparison among different studies.

\section{Conclusions}

At present apathy accompanying $\mathrm{AD}$ is apparently a disorder not largely investigated and its treatment still remains a challenge for pharmacotherapy. Only few treatments were proposed for apathy, but so far there is no clear demonstration of the advantage of one treatment versus others. Therapeutically, several small studies suggested a potential efficacy of various classes of compounds such as cholinesterase inhibitors, monoaminergic drugs, and other compounds with a heterogeneous pharmacological profile. The largest number of subjects was treated with cholinesterase inhibitors that at present may represent a possible option. Ginkgo biloba too, on the basis of published results, may be considered as a valid option. For other compounds the small size of samples and some concerns on safety make their role in relieving apathy of no practical importance.

Future research should be carried out using more rigid criteria compared to those used. The main needs are the use of randomized controlled trials and of the current diagnostic guidelines. New efficacy studies should include the evaluation of neuropsychological tests measuring the executive functions which are related to apathy and should also evaluate well-characterized patients, in which the disease severity and the cognitive measures are assessed and reported. Apathy, being one of the most frequent symptoms in $\mathrm{AD}$, needs to be further investigated and its treatment represents a priority.

\section{Conflict of Interests}

The authors declare that there is no conflict of interests regarding the publication of this paper.

\section{References}

[1] B. R. Stanton, P. N. Leigh, R. J. Howard, G. J. Barker, and R. G. Brown, "Behavioural and emotional symptoms of apathy are associated with distinct patterns of brain atrophy in neurodegenerative disorders," Journal of Neurology, vol. 260, no. 10, pp. 2481-2490, 2013.

[2] R. S. Marin, "Differential diagnosis and classification of apathy," American Journal of Psychiatry, vol. 147, no. 1, pp. 22-30, 1990.

[3] R. S. Marin, "Apathy: a neuropsychiatric syndrome," Journal of Neuropsychiatry and Clinical Neurosciences, vol. 3, no. 3, pp. 243-254, 1991.

[4] M. S. Mega, J. L. Cummings, S. Salloway, and P. Malloy, “The limbic system: an anatomic, phylogenetic, and clinical perspective," Journal of Neuropsychiatry and Clinical Neurosciences, vol. 9, no. 3, pp. 315-330, 1997.

[5] R. Levy and B. Dubois, "Apathy and the functional anatomy of the prefrontal cortex-basal ganglia circuits," Cerebral Cortex, vol. 16, no. 7, pp. 916-928, 2006.

[6] P. Tagariello, P. Girardi, and M. Amore, "Depression and apathy in dementia: same syndrome or different constructs? A critical review," Archives of Gerontology and Geriatrics, vol. 49, no. 2, pp. 246-249, 2009.

[7] A. M. Landes, S. D. Sperry, M. E. Strauss, and D. S. Geldmacher, "Apathy in Alzheimer's disease," Journal of the American Geriatrics Society, vol. 49, no. 12, pp. 1700-1707, 2001.

[8] P. H. Robert, E. Mulin, P. Malléa, and R. David, "Apathy diagnosis, assessment, and treatment in Alzheimer's disease," CNS Neuroscience and Therapeutics, vol. 16, no. 5, pp. 263-271, 2010.

[9] B. Winblad, G. B. Frisoni, L. Frolich et al., "EADC (European Alzheimer disease consortium) recommendations for future Alzheimer disease research in Europe," Journal of Nutrition, Health and Aging, vol. 12, no. 10, pp. 683-684, 2008.

[10] P. Robert, C. U. Onyike, A. F. G. Leentjens et al., "Proposed diagnostic criteria for apathy in Alzheimer's disease and other neuropsychiatric disorders," European Psychiatry, vol. 24, no. 2, pp. 98-104, 2009.

[11] Q. M. Samus, A. Rosenblatt, C. Steele et al., "The association of neuropsychiatric symptoms and environment with quality of life in assisted living residents with dementia," Gerontologist, vol. 45, no. 1, pp. 19-26, 2005.

[12] M. F. Weiner, L. S. Hynan, M. E. Bret, and C. White III, "Early behavioral symptoms and course of Alzheimer's disease," Acta Psychiatrica Scandinavica, vol. 111, no. 5, pp. 367-371, 2005.

[13] B. Seltzer, P. Zolnouni, M. Nunez et al., "Efficacy of donepezil in early-stage Alzheimer disease: a randomized placebocontrolled trial," Archives of Neurology, vol. 61, no. 12, pp. 18521856, 2004.

[14] M. Tanaka, C. Namiki, D. H. D. Thuy et al., "Prediction of psychiatric response to donepezil in patients with mild to moderate Alzheimer's disease," Journal of the Neurological Sciences, vol. 225, no. 1-2, pp. 135-141, 2004.

[15] K. Rockwood, S. Black, M.-A. Bedard, T. Tran, and I. Lussier, "Specific symptomatic changes following donepezil treatment of Alzheimer's disease: a multi-centre, primary care, open-label study," International Journal of Geriatric Psychiatry, vol. 22, no. 4, pp. 312-319, 2007.

[16] O. L. Lopez, J. A. Mackell, Y. Sun et al., "Effectiveness and safety of donepezil in hispanic patients with Alzheimer's disease: a 12-week open-label study," Journal of the National Medical Association, vol. 100, no. 11, pp. 1350-1358, 2008. 
[17] H. Brodaty, M. Woodward, K. Boundy, N. Barnes, and G. Allen, "A naturalistic study of galantamine for Alzheimer's disease," CNS Drugs, vol. 20, no. 11, pp. 935-943, 2006.

[18] M. S. Mega, I. D. Dinov, V. Porter et al., "Metabolic patterns associated with the clinical response to galantamine therapy: a fludeoxyglucose F18 positron emission tomographic study," Archives of Neurology, vol. 62, no. 5, pp. 721-728, 2005.

[19] S. Gauthier, A. Juby, W. Dalziel, B. Réhel, and R. Schecter, "Effects of rivastigmine on common symptomatology of Alzheimers disease (EXPLORE)," Current Medical Research and Opinion, vol. 26, no. 5, pp. 1149-1160, 2010.

[20] S. Gauthier, A. Juby, B. Rehel, and R. Schecter, "EXACT: rivastigmine improves the high prevalence of attention deficits and mood and behaviour symptoms in Alzheimer's disease," International Journal of Clinical Practice, vol. 61, no. 6, pp. 886$895,2007$.

[21] J. L. Cummings, B. Koumaras, M. Chen et al., "Effects of rivastigmine treatment on the neuropsychiatric and behavioral disturbances of nursing home residents with moderate to severe probable Alzheimer's disease: a 26-week, multicenter, OpenLabel Study," American Journal Geriatric Pharmacotherapy, vol. 3, no. 3, pp. 137-148, 2005.

[22] N. Herrmann, L. S. Rothenburg, S. E. Black et al., "Methylphenidate for the treatment of apathy in alzheimer disease: prediction of response using dextroamphetamine challenge," Journal of Clinical Psychopharmacology, vol. 28, no. 3, pp. 296-301, 2008.

[23] L. L. Frakey, S. Salloway, M. Buelow, and P. A. Malloy, "Randomized, double-blind, placebo-controlled trial of modafinil for the treatment of apathy in individuals with mild-to-moderate Alzheimer's disease," The Journal of Clinical Psychiatry, vol. 73, no. 6, pp. 796-801, 2012.

[24] P. R. Padala, W. J. Burke, V. K. Shostrom et al., "Methylphenidate for apathy and functional status in Dementia of the alzheimer type," American Journal of Geriatric Psychiatry, vol. 18, no. 4, pp. 371-374, 2010.

[25] A. Scripnikov, A. Khomenko, and O. Napryeyenko, "Effects of Ginkgo biloba extract EGb 761 on neuropsychiatric symptoms of dementia: findings from a randomised controlled trial," Wiener Medizinische Wochenschrift, vol. 157, no. 13-14, pp. 295-300, 2007.

[26] H. Siddique, L. S. Hynan, and M. F. Weiner, "Effect of a serotonin reuptake inhibitor on irritability, apathy, and psychotic symptoms in patients with Alzheimer's disease," Journal of Clinical Psychiatry, vol. 70, no. 6, pp. 915-918, 2009.

[27] G. A. Wells, B. Shea, D. O'Connell et al., The Newcastle-Ottawa Scale (NOS) for Assessing the Quality of Nonrandomised Studies in Meta-Analyses, Ottawa Hospital Research Institute, Ottawa, Canada, 2011.

[28] R. L. Drijgers, P. Aalten, A. Winogrodzka, F. R. J. Verhey, and A. F. G. Leentjens, "Pharmacological treatment of apathy in neurodegenerative diseases: a systematic review," Dementia and Geriatric Cognitive Disorders, vol. 28, no. 1, pp. 13-22, 2009. 


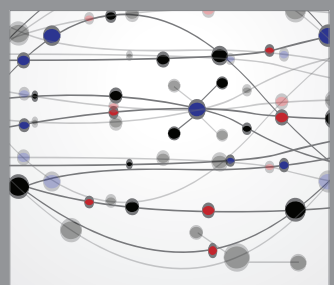

The Scientific World Journal
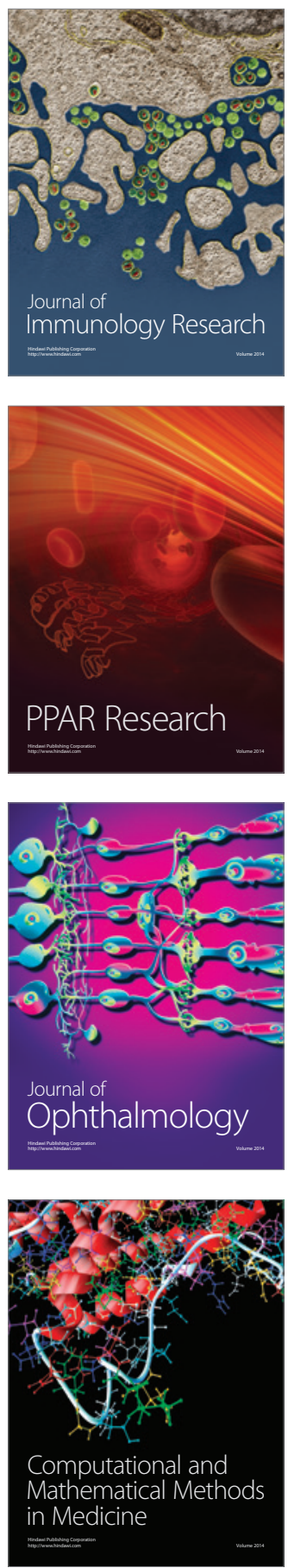

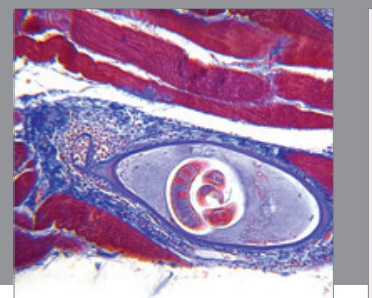

Gastroenterology

Research and Practice
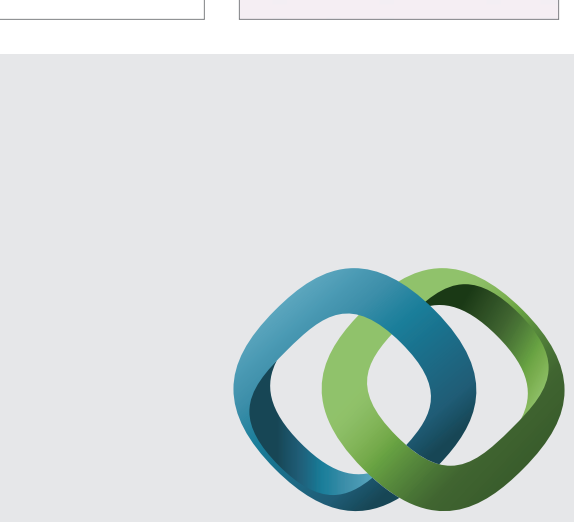

\section{Hindawi}

Submit your manuscripts at

http://www.hindawi.com
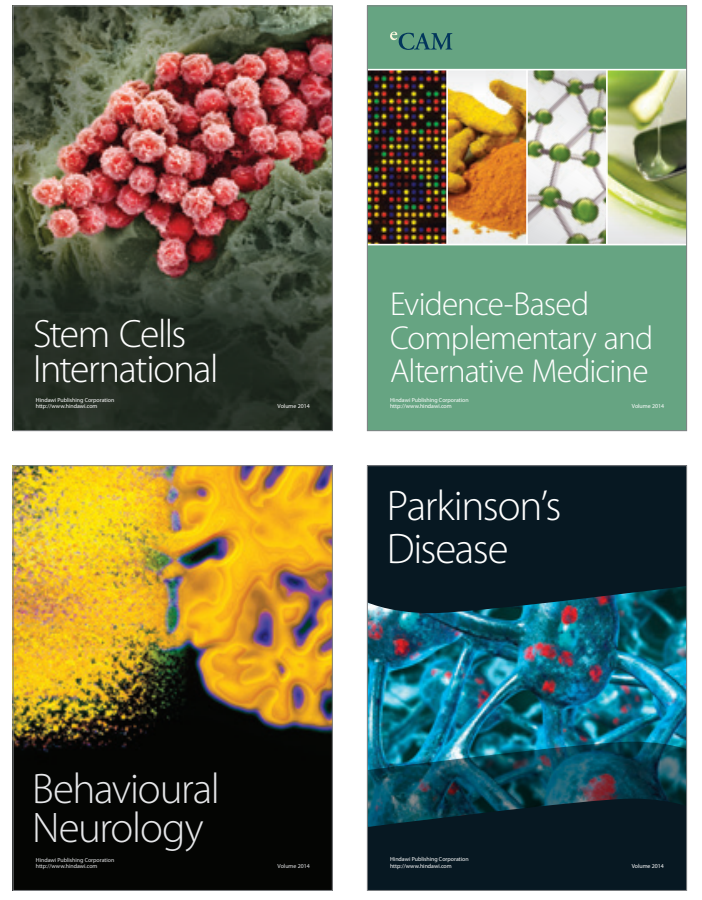
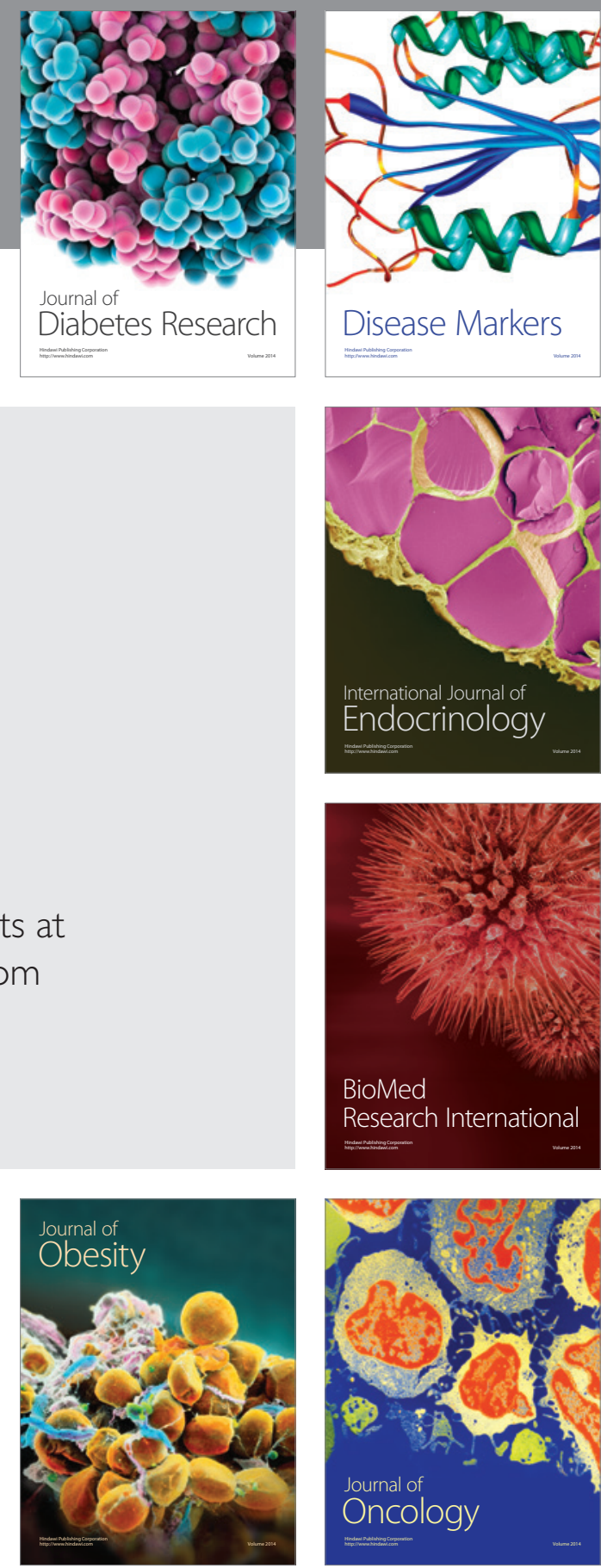

Disease Markers
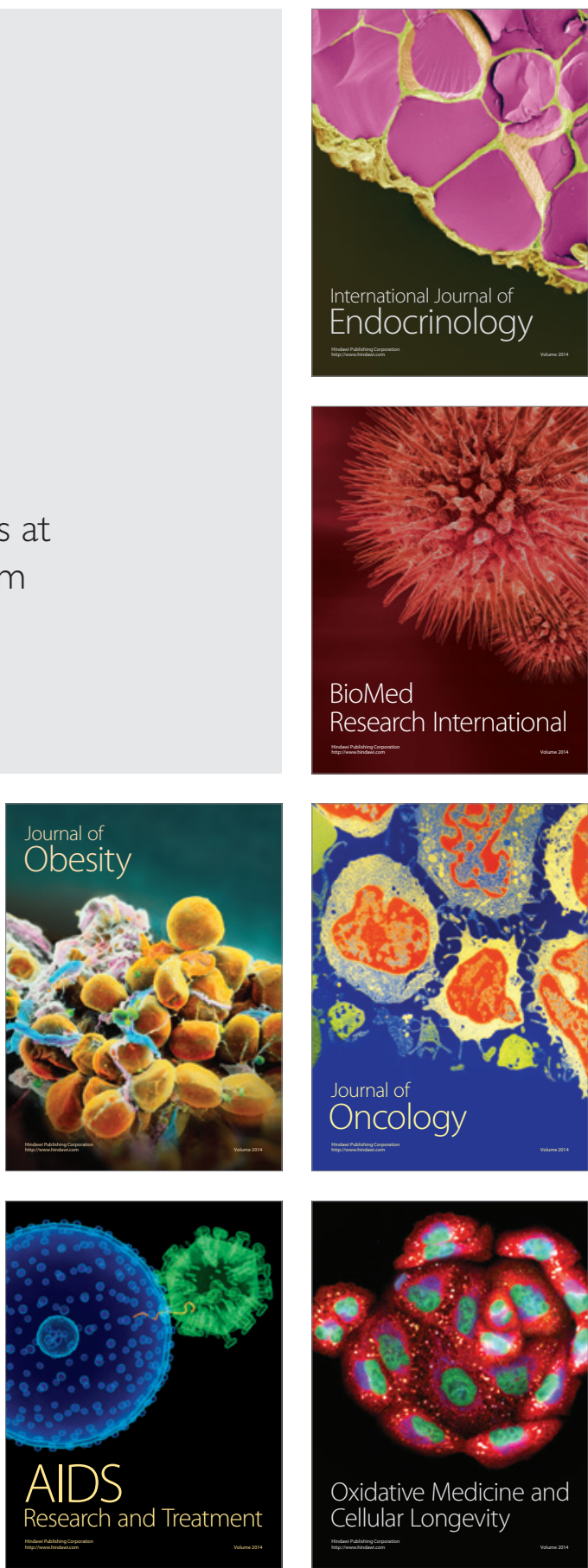